\title{
Unconditional Positive Self-Regard (UPSR) and Self-Compassion, the Internal Consistency and Convergent/Divergent Validity of Patterson \& Joseph's UPSR Scale
}

\author{
Laura Jayne Griffiths ${ }^{1}$, Christopher Alan Griffiths ${ }^{2}$ \\ ${ }^{1} \mathrm{NHS}$, Rugby, UK \\ ${ }^{2}$ Rethink Mental Illness, London, UK \\ Email: Laura.Griffiths@covwarkpt.nhs.uk
}

Received July 22, 2013; revised August 22, 2013; accepted August 30, 2013

\begin{abstract}
Copyright (C) 2013 Laura Jayne Griffiths, Christopher Alan Griffiths. This is an open access article distributed under the Creative Commons Attribution License, which permits unrestricted use, distribution, and reproduction in any medium, provided the original work is properly cited.
\end{abstract}

\begin{abstract}
Objectives: The purpose of this study is to test the psychometric properties and validity of the unconditional positive self-regard scale (UPSR) and its two subscales developed by Patterson \& Joseph (2006). It also aims to examine and compare the concepts of UPSR with self-compassion and its relation to mental well-being. Design: Correlation survey design validations of the UPSR scale (Patterson \& Joseph, 2006). Methods: The validation was conducted using an undergraduate and postgraduate student opportunity sample, $n=179$. Internal consistency was assessed using Cronbach's coefficient alpha and inter-item correlations. Convergent and divergent validity was explored in terms of correlations with self-compassion, depression (PHQ-9) and anxiety (GAD-7) scales. Results: There was good internal consistency for both the UPSR scale and the self-regard subscale and somewhat questionable internal consistency for the conditionality subscale. Overall the scale appears to be relatively consistent, supporting the previous findings reported by Patterson \& Joseph (2006). The results supported the hypothesis that UPSR is positively correlated with a measure of self-compassion and negatively correlated with measures of depression (PHQ-9) and anxiety (GAD-7). Conclusions: The UPSR scale is a valid measure of the person-centred concept of unconditional positive self-regard. This supports the potential use of the UPSR scale for evaluating therapeutic change for client-centred practitioners through the use of this non-medicalized tool.
\end{abstract}

Keywords: Unconditional Positive Self-Regard; Psychotherapy; Self-Compassion; Carl Rogers

\section{Introduction}

When individuals struggle to meet the demands of daily life and find that they need extra help, they can become self-critical and feel ashamed for not being able to cope [1]. Those individuals who have a tendency to be selfcritical are at a greater risk of psychopathology compared with individuals who self-reassure [2]. Individuals may try to fight their emotions, to "fix" themselves or try to avoid their negative feelings [1]. The emotions that are being avoided can become destructive, affecting mind, body and soul and can lead to further psychological distress [1,3]. Self-criticism and inner shame have a significant role in many forms of psychological disorder, including anxiety and depression [4,5]. An individual who experiences warm, empathic parenting with love and affection is more likely to be self-accepting and self-nurturing, and this is associated with good mental health [2].

A key aspect to consider when looking at self-processes is the person-centred construct of unconditional positive self-regard (UPSR). Carl Rogers $(1957 ; 2004)$ $[6,7]$, the founder of person-centred therapy developed a theory of personality that states that each individual has an inherent tendency to develop all of their capabilities in ways that serve to maintain or enhance self: he referred to this as the actualising tendency. Individuals come into psychological difficulty when they begin to internalise conditions of worth imposed on them from significant others, and the consequence of this is that their self-acceptance starts to become dependent on these often rigid 
internalised rules [8]. To highlight the negative impact that conditions of worth can have on an individual's psychological health, Assor, Roth \& Deci (2004) [9] investigated mothers' perceptions of their own parents use of conditional regard with them, and found that self-reported parental conditional regard was a predictor for poorer psychological well-being in the participants.

Rogers' person-centred personality theory suggests that psychological well-being involves individuals following their innate guidance mechanisms. According to Betz et al. (1995) [10] Rogers' client-centred therapy uses the concept of self-acceptance or unconditional selfregard as a goal for counselling to facilitate congruence between ones ideal self and actual self. The cause of psychological distress is argued to be the presence of a discrepancy between the individual's real and ideal selves [10]. Rogers (2004) [7] proposed that for a therapeutic situation to facilitate beneficial changes in the client, six necessary and sufficient therapeutic conditions need to be present. These include congruence, empathy and unconditional positive regard. Person-centred therapy aims to loosen these unyielding internalised rules and values which self-regard becomes contingent on, in order to have a positive effect on the client's psychological well-being [11].

Patterson \& Joseph (2006) [12] define UPSR as being a less stringent form of self-acceptance, and that an increase in UPSR can lead to a decrease in an individual's conditions of worth. Patterson \& Joseph's (2006) [12] work in this area considered the two-dimensional nature of the construct of UPSR that they state is implicit in Rogers' (1959) [11] definition and explicit in further work on this area. This consists of the positive self-regard aspect of UPSR (which Betz et al., 1995 [10], refers to as self-esteem) and also the unconditional aspect of UPSR, which according to Patterson \& Joseph [12] refers to the observation that self-regard can be conditional or unconditional. Patterson \& Joseph (2006) [12] argue that having higher levels of UPSR leads to a decrease in conditions of worth, and a loosening of the rigid internalised rules and values which self-regard becomes contingent on, thus leading to psychological well-being.

The construct of self-compassion is a Buddhist concept that in recent years has become the focus of research into new practices for creating mental well-being. It is introduced as an alternative to consider self-esteem when examining conceptualisations of having a healthy attitude and relationship to oneself $[13,14]$. Compared to selfesteem, self-compassion is believed to be a more effective route to happiness [1], with its non-evaluative emphasis [13] and with researchers showing evidence of individuals who are more self-compassionate tending to lead healthier and more productive lives than those individuals who are self-critical $[4,15]$. Self-compassion is defined as being able to treat oneself with kindness, recognising one's shared humanity and also being mindful when considering negative aspects of oneself [15]. Self-compassion involves encompassing emotional pain, and rather than fighting against it, responding with a kindness and understanding that we typically would give to others [1].

Neff (2003) [16] suggests that self-compassion consists of several elements, which include having a kind and non-judgemental attitude towards oneself during difficult times, recognising that each individual experience is part of a much larger, human experience, and also being able to have a balanced awareness of holding painful thoughts and feelings [17]. Self-compassion involves accepting painful thoughts and feelings without being judgemental or self-pitying $[14,16]$. To measure self-compassion, Neff (2003) [16] developed and validated her own scale, the Self-Compassion Scale. Selfcompassion has found to be significantly correlated with positive mental health outcomes that include a lack of depression, a lack of anxiety, emotional intelligence, social connectedness and greater life satisfaction $[15,16]$.

The importance for psychological therapies to have clear evidence base has become increasingly apparent $[18,19]$. The importance of evaluating therapeutic effectiveness has implications for funding decisions for the therapeutic provision in services [18]. Person-centred theory has often been criticised for lacking an empirical research base with its focus being on the whole person, not just specific symptoms (which are easily measurable); but Patterson \& Joseph (2006) [12] highlight how therapeutic changes in person-centred therapy can be evaluated with the use of non-medicalized evaluation tools that are theoretically grounded with person-centred theory and practice.

Tools should therefore measure effectiveness in terms of the outcomes that are predicted by the person-centred theory [12]. Rogers (1959) [11] states that his six necessary and sufficient core conditions are operationally definable and the changes that occur in therapy are measurable events. This therefore suggests that person-centred therapy is amenable to empirical evaluation, and it has a substantial empirical evidence base [20].

Patterson \& Joseph (2006) [12] developed a self-report measure of UPSR that is theoretically consistent with person-centred theory and therapy, and their initial findings have indicated its potential use as a tool for evaluating therapeutic change. It is a twelve-item scale comprising two separate subscales to account for the twodimensional nature of the construct of UPSR: self-regard and conditionality.

The UPSR scale (Patterson \& Joseph, 2006) [12] has not been independently validated since its initial development. This present study sets out to test validity. In seeking to do this the study examines the correlation between UPSR, self-compassion and mental illness (anxi- 
ety and depression). The first hypothesis is that the UPSR scale will be positively correlated with the SelfCompassion Scale, because they are believed to be theoretically similar. The based on the results of Betz et al.'s (1995) [10] UPSR study the second hypothesis is that the UPSR scale will be negatively correlated with the anxiety and depression scales.

\section{Method}

\subsection{Measures}

2.1.1. Unconditional Positive Self-Regard Scale [12] The Unconditional Positive Self-Regard (UPSR) scale is a twelve item measure scored on a five point Likert scale. The UPSR scale is composed of two separate subscales: self-regard (6 items on the scale, e.g. "I really value myself') and conditionality of positive self-regard (6 items on the scale, e.g. "Whether other people criticize me or praise me makes no difference to the way I feel about myself') [12]. The internal reliability of the main scale and the two subscales is adequate, with alpha coefficients all above 0.79 [12]. Comparison with other measures supported the validity of the scale. Patterson and Joseph (2006) [12] indicated that the UPSR scale may be a useful tool for the non-medicalised evaluation of therapeutic change.

\subsubsection{Self-Compassion Scale [16]}

The Self-Compassion Scale includes six subscales: SelfKindness (5 items, e.g., "I try to be loving towards myself when I'm feeling emotional pain"); Self-Judgment (5 items, e.g., "When I see aspects of myself that I don't like, I get down on myself"); Common Humanity (4 items, e.g., "When things are going badly for me, I see the difficulties as part of life that everyone goes through"); Isolation (4 items, e.g., "When I fail at something that's important to me, I tend to feel alone in my failure"); Mindfulness (4 items, e.g., "When I'm feeling down I try to approach my feelings with curiosity and openness"); and Over-Identification (4 items, e.g., "When something upsets me I get carried away with my feelings").

Using confirmatory factor analyses Neff (2003) [16] showed that a single higher-order factor of self-compassion explained the inter-correlations between the six subscales. Responses to the Self-Compassion Scale are completed using a 5-point Likert scale ranging from: "Almost Never" to "Almost Always", with higher scores representing greater self-compassion. The Self-Compassion Scale demonstrates concurrent validity, convergent validity, discriminant validity, and test-retest reliability [16,21]. This scale has demonstrated good internal consistency in various studies $(0.90$ - 0.95) [21].

\subsubsection{Patient Health Questionnaire (PHQ-9) [22]}

The PHQ-9 is a self-administered version of the Primary
Care Evaluation of Mental Disorders Patient Health Questionnaire (PRIME-MD) diagnostic instrument for common mental disorders, and is a 9-item depression scale. It is a widely used measure, for example it is routinely collected in all Improving Access to Psychotherapies (IAPT) services in the UK. It is described as being a powerful tool for assisting primary care clinicians in diagnosing depression (by assessing symptoms and functional impairment) as well as selecting and monitoring treatment. The respondent is required to rate each item out of $3(0=$ not at all, $1=$ several days, $2=$ more than half the days and 3 = nearly every day). Items include "Poor appetite or overeating?", "Feeling tired or having little energy?" and "Feeling down, depressed, or hopeless?"

Validity has been assessed against an independent structured mental health professional interview. PHQ-9 score $>10$ had a sensitivity of $88 \%$ and a specificity of $88 \%$ for major depression [23]. It has good internal consistency: Cronbach's alpha was reported at 0.89 [23].

\subsubsection{Generalized Anxiety Disorder (GAD-7) [24]}

The GAD-7 is also a self-report tool and consists of seven short items and is used to screen severity in both clinical practice and research. It is a widely used measure, for example it is routinely collected in all IAPT services in the UK. The respondent is required to rate each item out of $3(0=$ not at all, $1=$ several days, $2=$ more than half the days and $3=$ nearly every day). Items include "Trouble relaxing", "Feeling nervous, anxious or on edge" and "Feeling afraid as if something awful might happen". A score that is rated as severe on this scale is between 16 and 20 .

When used as a screening tool, further evaluation is recommended when the score is 10 or greater. Using the threshold of 10 , the GAD-7 has a sensitivity of $89 \%$ and a specificity of $82 \%$ for generalised anxiety disorder. It is moderately good at screening three other common anxiety disorders - panic disorder (sensitivity 74\%, specificity $81 \%$ ), social anxiety disorder (sensitivity $72 \%$, specificity $80 \%$ ) and post-traumatic stress disorder (sensitivity $66 \%$, specificity $81 \%$ ) [25]. It has good internal consistency: Cronbach's alpha is reported to be 0.92 [25].

\subsection{Participants}

A total of 179 university undergraduate and post graduate students completed the questionnaire. The sample included 29 males and 150 females. The average age of the participants was 31 years (standard deviation = 11.26). The youngest participant was 18 years old and the oldest was 67 years old (Table 1).

\subsection{Procedure}

The survey was administered to a convenience sample of 
Table 1. Percentage of participants per age category.

\begin{tabular}{cc}
\hline Age category & Percentage of participants \\
\hline $18-25$ & $40.8 \%$ \\
$26-33$ & $26.8 \%$ \\
$34-41$ & $9.5 \%$ \\
$42-49$ & $14.6 \%$ \\
$50-57$ & $6.9 \%$ \\
$58-65$ & $1.1 \%$ \\
$65+$ & $0.6 \%$ \\
\hline
\end{tabular}

students attending a UK university. Consent was gained through agreeing to complete the scales. The participants completed the survey online. Students were asked to provide information on age and sex before completing the measures. The measures were completed in the following order: UPSR scale, Self-Compassion Scale, GAD-7 and PHQ-9. Only data where all scales were fully completed were used. A critical value of $p<0.01$ was adopted in order to control for Type 1 error.

\section{Results}

\subsection{Means and Medians}

The mean scores were UPSR full-scale $=34$, conditionality subscale $=14$, and self-regard subscale $=20$. The UPSR scale median score was 34 , the conditionality subscale median score was 14 , and the self-regard subscale median score was 21 .

\subsection{Internal Consistency}

The standardised Cronbach's coefficient alpha of the UPSR scale was 0.806 and the self-regard subscale was 0.898 , both good internal consistency falling well above the recommended lower limit (scores above 0.7 indicate good internal consistency) [26]. The standardised Cronbach's alpha of the conditionality subscale was 0.661 which indicates somewhat questionable internal consistency. The two subscales were shown to have weak positive inter-correlation, $r=0.258(\mathrm{p}<0.01)$, indicating low shared variance between the scales.

Pearson's product moment correlation coefficient (r) was used to assess the UPSR scales inter-item correlations, and these ranged from -0.148 to 0.662 . The mean score was found to be 0.257 . The conditionality subscale inter-item correlations ranged from 0.028 to 0.765 with a mean score of 0.245 . All items of the self-regard subscale had inter-item correlations above 0.5 with a range of 0.509 to 0.662 with a mean score of 0.595 . This supports the validity of the self-regard subscale, as all of these correlations are within the desired limits.

\subsection{Convergent Validity}

Pearson's product moment correlation coefficient (r) was conducted to examine the relationship between the UPSR scale and its two subscales, with the Self-Compassion Scale. The UPSR scale and its two subscales were positively correlated with the Self-Compassion Scale. The Self-Compassion Scale showed a strong significant correlation with UPSR $(r=0.644, p<0.01)$ and self-regard $(\mathrm{r}=0.622, \mathrm{p}<0.01)$ and moderate significant correlation with conditionality $(r=0.374, p<0.01)$.

\subsection{Divergent Validity}

This was assessed using Pearson's product moment correlation coefficient ( $r$ ) to explore the relationships between the total UPSR scale, and also its two subscales, with a scale of depression (PHQ-9) and a scale of anxiety (GAD-7). The UPSR scale and its two subscales were negatively correlated with depression. Depression (PHQ9) showed a moderate significant negative correlation with UPSR $(r=-0.455, p<0.01)$ and a strong significant negative correlation with self-regard $(\mathrm{r}=-0.527, \mathrm{p}<$ $0.01)$ and weak significant negative correlation with conditionality $(\mathrm{r}=-0.150, \mathrm{p}<0.01)$.

The UPSR scale and its two subscales were negatively correlated with anxiety. Anxiety (GAD-7) showed a moderate significant negative correlation with the UPSR scale $(\mathrm{r}=-0.427, \mathrm{p}<0.01)$ and self-regard subscale $(\mathrm{r}=$ $-0.431, \mathrm{p}<0.01)$ and a weak significant negative correlation with the conditionality subscale $(\mathrm{r}=-0.222, \mathrm{p}<$ 0.01) (Table 2).

The mean scores on all of the measure were split by sex and are listed in Table 3. T-tests revealed that there was no significant difference between men and women in scores on any of the measures (Table 3).

\section{Discussion}

The results of the Cronbach's coefficient alpha analysis showed that there was good internal consistency for both the UPSR full-scale score and the self-regard subscale, as both scores were higher than a set minimum level [26]. This is consistent with the Cronbach's coefficient alpha

Table 2. Correlations of the UPSR full scale and its subscales of self-regard and conditionality, thee Self-Compassion Scale [15], the Generalised Anxiety Disorder (GAD-7) scale and the Patient Health Questionnaire (PHQ-9) (measuring depression).

\begin{tabular}{lccc}
\hline & Self-Compassion Scale & GAD-7 & PHQ-9 \\
\hline UPSR & 0.644 & -0.427 & -0.455 \\
Self-regard subscale & 0.622 & -0.431 & -0.527 \\
Conditionality subscale & 0.374 & -0.222 & -0.150 \\
\hline
\end{tabular}


Table 3. Mean scores of the UPSR full scale, the self-regard and conditionality subscales of the UPSR, the Self-Compassion Scale, the anxiety scale and the depression scale, by sex.

\begin{tabular}{ccc}
\hline Measure & Sex & Mean \\
\hline UPSR & Male & 36.21 \\
& Female & 34.16 \\
Self-regard & Male & 20.9 \\
& Female & 19.88 \\
Conditionality & Male & 15.31 \\
& Female & 14.23 \\
Self-compassion & Male & 76.03 \\
& Female & 72.55 \\
Anxiety & Male & 13.59 \\
& Female & 13.93 \\
Depression & Male & 15.17 \\
& Female & 15.58 \\
\hline
\end{tabular}

scores reported in the original study [12]. The results of the Cronbach coefficient alpha analysis for the conditionality subscale however showed questionable internal validity as this was lower than the desired level. It was also lower than the reported level by Patterson \& Joseph (2006) [12]. However, despite this finding, the overall results suggest that the overall scale is relatively consistent.

The tests of internal consistency of this present study supported Patterson and Joseph's [12] findings that the UPSR scale consists of two subscales: self-regard and conditionality. Inter-item correlations of the UPSR scale showed that some of the items had a negative inter-item correlation, which differs to the original study which found all item-total correlations to be above 0.30 . The self-regard subscale is shown to be particularly robust, with all inter-item correlations being above 0.5 . It is clear that the self-regard subscale items appear to be a stronger indicator of the presence or absence of this construct in the participants who completed this study.

The results of this present study support the convergent validity of the UPSR scale as a strong positive correlation with Self-Compassion Scale [16] was found, and which further supports the validity of this scale. Divergent validity was demonstrated through a significant negative correlation between scores on the UPSR scale and scores on measures of anxiety and depression, providing further support for the validity of the UPSR measure.

Considering the correlation between the two subscales, the results indicate some shared variances between the items. These current results suggest that there is a sig- nificant relationship, although one that is weak, but in a positive direction. This would support the use of the total score of the two scales as being an overall single measure. Considering the subscales individually has shown that the self-regard subscale is a robust measure that may perhaps be useful on its own and may be a stronger indicator of the presence or absence of self-regard in individuals. The findings for the conditionality subscale differ slightly to the findings in the original study. The construct suggests then that the conditionality subscale may provide information about a different aspect of relating to self than what has been considered in the literature.

An association between the concept of UPSR and psychopathology is clear in these results. In line with Rogers theory (1959) [11] placing internal conditions of worth on oneself, or making self-regard be contingent on certain conditions (for example, relying on the praise of others) correlates with psychopathology (anxiety and depression in this study). These findings support the overall findings of Patterson \& Joseph (2006) [12] for the use of this scale as a non-medicalized indicator of change in person-centred therapy.

Another finding is the association between the SelfCompassion Scale and the UPSR scale. Both scales appear to be related measures of self-esteem, which indicates that there could be several ways of conceptualising self-esteem. Research could be developed in this area to further our understanding of self-esteem. There is a lack of validated measures of the different conceptualisations of self-esteem and so the findings here are important for this research area as the UPSR offers an alternative measure.

There was no significant difference between men and women in scores found on any of the measures in this current study. Patterson \& Joseph (2006) [12] did not report these figures. Scores on the anxiety and depression scales were not consistent with previous findings that women score significantly higher on anxiety and depression [27]. In relation to self-compassion this study's result opposes that of Neff (2003) [16] who found that women reported having significantly less self-compassion than men. Therefore, the results are not consistent with past findings that females tend to be more critical of themselves and tend to ruminate on their negative feelings more than males do (Leadbeater et al., 1999; NolenHoeksema et al., 1999 cited in [13]). However, they are consistent with findings that women and men are equally kind and gentle to themselves and see their experiences as part of common humanity [16].

There are a number of limitations to the results of the study. There were more women than men in the sample thus limiting the generalizability of the results to men. The sample was a non-clinical opportunity sample made up of university students, which limits the generalizabil- 
ity to other population groups. However, there was a higher average age than for many student population studies; nevertheless, the majority of the participants were aged 18 - 33 years $(67.6 \%)$ which also limits the generalizability of the results to those over 33 years old.

\section{Conclusion}

The main aim of the paper was to access the validity of the UPSR scale and the results of the convergent and divergent correlations, plus the tests of internal consistency offers support for the validity and psychometric properties of the UPSR scale. It supports the overall findings of Patterson \& Joseph's (2006) [12] study. Further validation could employ other convergent/divergent measures. The brevity of the UPSR measure is likely to be a valued attribute as an evaluative tool for therapeutic outcomes when it comes to assessing unconditional positive self-regard.

\section{REFERENCES}

[1] C. K. Germer, "The Mindful Path to Self-Compassion: Freeing Yourself from Destructive Thoughts and Emotions," The Guilford Press, New York, 2009.

[2] P. Gilbert, M. W. Baldwin, C. Irons, J. R. Baccus and M. Palmer, "Self-Criticism and Self-Warmth: An Imagery Study Exploring Their Relation to Depression," Journal of Cognitive Psychology: An International Quarterly, Vol. 20, No. 2, 2006, pp. 183-200.

[3] P. Gilbert, "Compassion: Conceptualisations, Research and Use in Psychotherapy," Routledge, Sussex, 2009.

[4] P. Gilbert, M. W. Baldwin, C. Irons, J. R. Baccus and M. Palmer, "Self-Criticism and Self-Warmth: An Imagery Study Exploring Their Relation to Depression," Journal of Cognitive Psychology: An International Quarterly, Vol. 20, No. 2, 2006, pp. 183-200.

[5] N. B. Allen and W. E. J. Knight, "Mindfulness, Compassion for Self, and Compassion for Others: Implications for Understanding the Psychopathology and Treatment of Depression,” In: P. Gilbert, Ed., Compassion: Conceptualisations, Research and Use in Psychotherapy, Routledge, London, 2009, pp. 239-262.

[6] C. R. Rogers, "The Necessary and Sufficient Conditions of Therapeutic Personality Change," Journal of Consulting Psychology, Vol. 21, No. 1, 1957, pp. 95-103. http://dx.doi.org/10.1037/h0045357

[7] C. R. Rogers, "Way of Being," Houghton Mifflin, Boston, 2004.

[8] S. Joseph and D. Murphy, "Person-Centred Approach, Positive Psychology, and Relational Helping: Building Bridges," Journal of Humanistic Psychology, Vol. 53, No. 1, 2013, pp. 26-51. http://dx.doi.org/10.1177/0022167812436426

[9] A. Assor, G. Roth and E. L. Deci, "The Emotional Costs of Parents' Conditional Regard: A Self-Determination Theory Analysis," Journal of Personality, Vol. 72, No. 1,
2004, pp. 42-88. http://dx.doi.org/10.1111/j.0022-3506.2004.00256.x

[10] N. E. Betz, E. Wohlgemuth, D. Serling, J. Harshbargerand and K. Klein, "Evaluation of a Measure of Self-Esteem Based on the Concept of Unconditional Self-Regard," Journal of Counseling and Development, Vol. 74, No. 1, 1995, pp. 76-82. http://dx.doi.org/10.1002/j.1556-6676.1995.tb01826.x

[11] C. R. Rogers, "A Theory of Therapy, Personality, and Interpersonal Relationships as Developed in the ClientCentred Framework," In: S. Koch Ed., Psychology: A Study of a Science, Formulations of the Person and the Social Context, McGraw-Hill, New York, 1959, pp. 184256.

[12] T. G. Patterson and S. Joseph, "Development of a SelfReport Measure of Unconditional Positive Self-Regard," Psychology and Psychotherapy: Theory, Research, and Practice, Vol. 79, No. 4, 2006, pp. 557-570.

http://dx.doi.org/10.1348/147608305X89414

[13] K. D. Neff, "Self-Compassion: An Alternative Conceptualization of a Healthy Attitude toward Oneself," Self and Identity, Vol. 2, No. 2, 2003, pp. 85-101. http://dx.doi.org/10.1080/15298860309032

[14] K. D. Neff, K. L. Kirkpatrick and S. S. Rude. "Self-Compassion and Adaptive Psychological Functioning," Journal of Research in Personality, Vol. 41, No. 1, 2007, pp. 139-154. http://dx.doi.org/10.1016/j.jrp.2006.03.004

[15] K. D. Neff and R. Vonk, "Self-Compassion versus Global Self-Esteem: Two Different Ways of Relating to Oneself," Journal of Personality, Vol. 77, No. 1, 2009, pp. 23-50.

http://dx.doi.org/10.1111/j.1467-6494.2008.00537.x

[16] K. D. Neff, "Development and Validation of a Scale to Measure Self-Compassion," Self and Identity, Vol. 2, No. 3, 2003, pp. 223-250. http://dx.doi.org/10.1080/15298860309027

[17] R. A. Baer, G. T. Smith, J. Hopkins, J. Krietemeyer and L. Toney, "Using Self-Report Assessment Methods to Explore Facets of Mindfulness," Assessment, Vol. 13, No. 1, 2006, pp. 27-45. http://dx.doi.org/10.1177/1073191105283504

[18] J. Deegear and D. M. Lawson, "The Utility of Empirically Supported Treatments," Professional Psychology: Research and Practice, Vol. 34, No. 3, 2003, pp. 271-277. http://dx.doi.org/10.1037/0735-7028.34.3.271

[19] D. Sue and D. M. Sue, "Foundations of Counseling and Psychotherapy," John Wiley \& Sons, New Jersey, 2008.

[20] T. G. Patterson and S. Joseph, "Person-Centred Personality Theory: Support from Self-Determination Theory and Positive Psychology," Journal of Humanistic Psychology, Vol. 47, No. 1, 2007, pp. 117-139. http://dx.doi.org/10.1177/0022167806293008

[21] K. D. Neff and P. McGehee, "Self-Compassion and Psychological Resilience among Adolescents and Young Adults. Self and Identity," Vol. 9, No. 3, 2010, pp. 225240. http://dx.doi.org/10.1080/15298860902979307

[22] R. L. Spitzer, K. Kroenke, J. B. W. Williams and the Patient Health Questionnaire Primary Care Study Group, 
"Validation and Utility of a Self-Report Version of PRIME-MD. The PHQ Primary Care Study," The Journal of the American Medical Association, Vol. 282, No. 18, 1999, pp. 1737-1744.

http://dx.doi.org/10.1001/jama.282.18.1737

[23] K. Kroenke, R. L. Spitzer and J. B. W. Williams, "The PHQ-9: Validity of a Brief Depression Severity Measure," Journal of General Internal Medicine, Vol. 16, No. 9, 2001, pp. 606-613.

http://dx.doi.org/10.1046/j.1525-1497.2001.016009606.x

[24] R. L. Spitzer, K. Kroenke, J. B. W. Williams and B. Löwe, "A Brief Measure for Assessing General Anxiety Disorder: The GAD-7," Archives of Internal Medicine, 166, No. 10, 2006, pp. 1092-1097.

http://dx.doi.org/10.1001/archinte.166.10.1092
[25] K. Kroenke, R. L. Spitzer and J. B. W. Williams, P. O., Monahan and B. Löwe, "Anxiety Disorders in Primary Care: Prevalence, Impairment, Comorbidity, and Detection," Annuals of Internal Medicine, Vol. 146, No. 5, 2007, pp. 317-325.

http://dx.doi.org/10.7326/0003-4819-146-5-200703060-0 $\underline{0004}$

[26] J. Pallant, "SPSS Survival Manual," 4th Edition, Open University Press, Berkshire, 2010.

[27] B. Löwe, O. Decker, S. Müller, E. Brähler, D. Schellberg, W. Herzog and P. Y. Herzberg, "Validation and Standardization of the Generalized Anxiety Disorder Screener (GAD-7) in the General Population," Medical Care, Vol. 46, No. 3, 2008, pp. 266-274. http://dx.doi.org/10.1097/MLR.0b013e318160d093 